\title{
Safety of a Scout Dose Preceding Hepatic Radioembolization with ${ }^{166} \mathrm{Ho}$ Microspheres
}

\author{
Jip F. Prince, Rob van Rooij, Gijsbert H. Bol, Hugo W.A.M. de Jong, Maurice A.A.J. van den Bosch, \\ and Marnix G.E.H. Lam \\ Department of Radiology and Nuclear Medicine, University Medical Center Utrecht, Utrecht, The Netherlands
}

\begin{abstract}
Before ${ }^{166} \mathrm{Ho}$ radioembolization, a small batch of the same type of microspheres is administered as a scout dose instead of the conventional ${ }^{99 m}$ Tc-macroaggregated albumin ( ${ }^{99 m T c-M A A) . ~ T h e ~}{ }^{166} \mathrm{Ho}$ scout dose provides a more accurate and precise lung shunt assessment. However, in contrast to ${ }^{99 m} \mathrm{Tc}-\mathrm{MAA}$, an unintended extrahepatic deposition of this $\beta$-emitting scout dose could inflict radiation damage, the extent of which we aimed to quantify in this study. Methods: All patients eligible for radioembolization in our institute between January 2011 and March 2014 were reviewed. Of the extrahepatic depositions of 99mTC-MAA on SPECT, the amount and volume were measured. These were used to calculate the theoretic absorbed dose in the case a ${ }^{166} \mathrm{Ho}$ scout dose had been used. The extrahepatic activity was measured as the sum of all voxels of the deposition. Volumes were measured using a threshold technique including all voxels from the maximum voxel intensity up to a certain percentage. The threshold needed to obtain the true volume was studied in a phantom study. Results: In the phantom study, a threshold of $40 \%$ was found to overestimate the volume, with the consequence of underestimating the absorbed dose. Of 160 patients, 32 patients (34 cases) of extrahepatic deposition were identified. The depositions contained a median of $1.3 \%$ (range, $0.1 \%-19.5 \%$ ) of the administered activity in a median volume of $6.8 \mathrm{~mL}$ (range, 1.1$42 \mathrm{~mL}$ ). The use of a scout dose of $250 \mathrm{MBq}$ of ${ }^{166} \mathrm{Ho}$ microspheres in these cases would theoretically have resulted in a median absorbed dose of 6.0 Gy (range, 0.9-374 Gy). The dose exceeded a limit of 49 Gy (reported in 2013) in 2 of 34 cases (5.9\%; 95\% confidence interval, $0.7 \%-20.1 \%)$ or 2 of 160 (1.3\%; $95 \%$ confidence interval, $0.1 \%-4.7 \%$ ) of all patients. In these 2 patients with a large absorbed dose (112 and $374 \mathrm{~Gy}$ ), the culprit vessel was identified in 1 case. Conclusion: Extrahepatic deposition of a ${ }^{166} \mathrm{Ho}$ scout dose seems to be theoretically safe in most patients. Its safety in clinical practice is being evaluated in ongoing clinical trials.
\end{abstract}

Key Words: interventional oncology; radioembolization; ${ }^{99 m T C-M A A ; ~}$ holmium

J Nucl Med 2015; 56:817-823

DOI: 10.2967/jnumed.115.155564

$\mathbf{R}$ adioembolization is a minimally invasive treatment for hepatic malignancies. Millions of radioactive microspheres are injected in the

Received Feb. 6, 2015; revision accepted Apr. 15, 2015.

For correspondence or reprints contact: Jip F. Prince, University Medical Center Utrecht, Department of Radiology and Nuclear Medicine, Heidelberglaan 100, 3584 CX Utrecht, The Netherlands.

E-mail: jipfprince@gmail.com

Published online Apr. 30, 2015.

COPYRIGHT (c) 2015 by the Society of Nuclear Medicine and Molecular Imaging, Inc. hepatic artery to radiate and embolize malignancies (1). Deposition of microspheres in gastrointestinal organs can result in ulceration or inflammation of tissue by a combination of embolization and radiation damage (2-4). To prevent this, vessels leading to gastrointestinal organs may be coil-embolized in patients scheduled for radioembolization treatment, during a pretreatment session using contrastenhanced images (i.e., digital subtraction angiography complemented

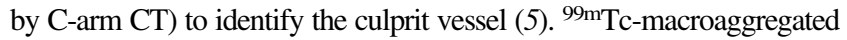
albumin ( ${ }^{99}$ Tc-MAA) is administered in this pretreatment session to simulate intrahepatic treatment biodistribution and to assess lung shunting and the possibility of extrahepatic deposition of the microspheres (6). This procedure is safe because ${ }^{99 \mathrm{~m} T c-M A A}$ emits $140-\mathrm{keV} \gamma$ photons (detectable by scintigraphy), which deposit a negligible dose, and the macroaggregated albumin dissolves in the bloodstream in several hours.

Treatment with ${ }^{166} \mathrm{Ho}$ radioembolization can be preceded by a different scout dose used during the pretreatment session (7). This scout dose consists of a small batch of microspheres (250 MBq), identical to the treatment ${ }^{166} \mathrm{Ho}$ microspheres. It is expected to better simulate treatment because it shares the same density, size distribution, and morphology with the treatment dose. It was already shown that a ${ }^{166} \mathrm{Ho}$ scout dose was a more reliable predictor of lung shunting than ${ }^{99 m}$ Tc-MAA (8). However, this scout dose emits electrons due to its $\beta^{-}$decay. In the case of unwanted extrahepatic deposition of activity, a ${ }^{166} \mathrm{Ho}$ scout dose may induce ulceration and inflammation of abdominal organs, such as the stomach, duodenum, or pancreas. In prior studies on ${ }^{166} \mathrm{Ho}$ radioembolization of the liver, both ${ }^{99 \mathrm{~m}} \mathrm{Tc}-$ MAA and a ${ }^{166} \mathrm{Ho}$ scout dose preceded treatment (7). In order to replace ${ }^{99 \mathrm{~m}} \mathrm{Tc}-\mathrm{MAA}$ in clinical practice, the risk of extrahepatic deposition of a ${ }^{166} \mathrm{Ho}$ scout dose must be known.

This study uses extrahepatic deposition of ${ }^{99 \mathrm{~m}} \mathrm{Tc}-\mathrm{MAA}$ as a surrogate marker for an extrahepatic scout dose, because the ${ }^{166} \mathrm{Ho}$ scout dose, used in the Holmium Embolization Particles for Arterial Radiotherapy (HEPAR) trials, was preceded by ${ }^{99 m}$ Tc-MAA injection, which decreased the incidence of extrahepatic deposition to negligible figures. The objective of this study was to calculate the theoretic absorbed dose of extrahepatic deposition from a ${ }^{166} \mathrm{Ho}$ scout dose by evaluation of the extrahepatic depositions of ${ }^{99 m}$ Tc-MAA as seen in current practice. A phantom study was performed to determine the volume of the depositions on SPECT.

\section{MATERIALS AND METHODS}

\section{Study Design}

In this retrospective cross-sectional study, all pretreatment procedures before radioembolization were reviewed for extrahepatic deposition of ${ }^{99 \mathrm{~m}} \mathrm{Tc}-\mathrm{MAA}$. From these procedures, the amount and volume of the deposition were estimated. The partial-volume effect makes absolute quantification of focal uptake challenging, as is known from, 
for example, analysis of standardized uptake values from ${ }^{18} \mathrm{~F}$-FDG PET (9). Therefore, likewise as is practice in ${ }^{18} \mathrm{~F}-\mathrm{FDG}$ PET, the technique used for volume estimation was first tested in a phantom study. Using the amount and volume, we calculated the potential absorbed dose in the case that a $250-\mathrm{MBq}{ }^{166} \mathrm{Ho}$ scout dose would have been used instead of a $150-\mathrm{MBq}{ }^{99 \mathrm{~m}} \mathrm{Tc}-\mathrm{MAA}$ dose. A waiver for informed consent for the retrospective review of these imaging data was obtained from the research ethics committee of our institution.

\section{Phantom Equipment}

For evaluation of the volume-measurement method, the National Electrical Manufacturers Association NU2-2001 image-quality phantom, containing 6 spheres of sizes varying between 0.5 and $26.5 \mathrm{~mL}$ suspended in a water-filled background compartment of $9.7 \mathrm{~L}$, was used. The spheres were filled from a ${ }^{99 \mathrm{~m}} \mathrm{Tc}$ buffer solution of known activity concentration and scanned identically to the ${ }^{99 \mathrm{~m} T c-M A A}$ patient protocol.

\section{Patient Population}

All consecutive patients from January 2011 (SPECT/CT routinely performed since then) until March 2014, who received a ${ }^{99 m}$ Tc-MAA injection as part of radioembolization workup, were eligible for inclusion. To obtain a representative sample of the distribution of extrahepatic depositions, if a patient underwent multiple angiography sessions, only the first was included (i.e., if an extrahepatic deposition is corrected in a subsequent angiography it should either disappear or become smaller; the second angiography session probably results in smaller depositions than the first). If 2 separate extrahepatic depositions were in a single patient, they were evaluated as 2 separate cases.

\section{Procedure}

Every patient underwent pretreatment angiography together with injection of $150 \mathrm{MBq}$ of ${ }^{99 \mathrm{~m}} \mathrm{Tc}-\mathrm{MAA}(0.8 \mathrm{mg}$, Technescan LyoMAA [Mallinckrodt Medical B.V.]) according to published guidelines (10). The gastroduodenal or gastric artery were coil-embolized at the discretion of the interventional radiologists; some preferred to always occlude those arteries whereas others injected ${ }^{99} \mathrm{~m}$ Tc-MAA in a selective, lobar, fashion. The operator confirmed the injection position using dual-subtraction angiography, complemented by $\mathrm{C}$-arm CT. Patients were planned to receive either yttrium resin or holmium microspheres as a whole-liver or lobar treatment.

\section{Imaging and Reconstruction}

All patients were scanned on a Symbia T16 SPECT/CT scanner (Siemens) within an hour after the pretreatment angiography with injection of ${ }^{99 m}$ Tc-MAA. SPECT data of the liver were acquired using a low-energy high-resolution collimator, on a $128 \times 128$ matrix (zoom, 1.23; pixel size, $3.9 \times 3.9 \mathrm{~mm}$ ) with 120 angles $(20 \mathrm{~s}$ per projection) over a noncircular $360^{\circ}$ orbit and a $140-\mathrm{keV} \pm 7.5 \%$ photopeak energy window. Low-dose CT data $(110 \mathrm{kVp}, 40 \mathrm{mAs}$, adaptive dose modulation with Siemens CARE Dose 4D) were acquired and reconstructed to a voxel size of $1.27 \times 1.27 \times 5 \mathrm{~mm}$ using a smoothing kernel (B08s; Siemens Healthcare). After a CT-derived attenuation map was created (Syngo MI Applications; Siemens Healthcare), SPECT images were reconstructed using 3-dimensional orderedsubset expectation maximization (Flash 3D; Siemens) with 6 iterations, 8 subsets; $5 \mathrm{~mm}$ gaussian smoothing; CT-based attenuation correction; and a window-based scatter correction.

\section{Analysis of Deposition: Activity and Volume}

The extrahepatic activity was estimated by summation of all voxels within a manual delineation of the deposition. It was delineated using in-house-developed software (Volumetool) (11). The margin of delineation was large enough to visually include all focal activity including the displaced counts due to resolution, patient motion, and scatter effects. If the counts in the liver and the displaced counts from the deposition overlapped, the point of the lowest intensity was assumed to be the best estimate of the boundary between the 2. All voxels within the volume were summed, without use of a threshold, to prevent underestimation of the activity (and absorbed dose). The total administered activity was estimated by a delineation around the liver, which included the deposition but excluded the kidneys, thyroid, stomach, and lungs, because the microspheres used in radioembolization do not shunt to extrahepatic organs in the same way as free pertechnetate from ${ }^{99 \mathrm{~m}}$ Tc-MAA (12). The percentage of counts in the deposition relative to the counts in the liver (and deposition) was used to predict the activity in the deposition if ${ }^{166} \mathrm{Ho}$ were to be used as a scout dose.

To determine the volume of an extrahepatic deposition, the limited resolution of the SPECT image needed to be accounted for. As a start, the same manual delineation of the deposition as mentioned before was used, which included the extrahepatic deposition but excluded the liver. Subsequently, the volume was estimated by selecting only voxels within the start delineation exceeding a certain threshold determined in the phantom study (a percentage of the maximum value in the delineation). These voxels were considered to be contributing to the volume; the voxels with lower values were considered to be a result of the point-spread function of the SPECT. The percentage was determined in a phantom study to provide a conservative underestimation of the volume, which would result in an overestimation of the absorbed dose.

\section{Absorbed Dose}

For theoretic assessment of the potential risk of extrahepatic ${ }^{166} \mathrm{Ho}$ microsphere deposition, the ${ }^{99 \mathrm{~m}} \mathrm{Tc}-\mathrm{MAA}$ was assumed to be distributed identically to ${ }^{166} \mathrm{Ho}$ microspheres with regard to the amount and volume of extrahepatic activity.

No adjustments to the prescribed activities were made for high lung shunt fractions. Patients who had contraindications for treatment were not excluded from this analysis. The following formula was used to calculate the dose:

$$
\begin{aligned}
& \text { Dose }(\mathrm{Gy})= \\
& \qquad 15.87\left(\frac{\mathrm{mJ}}{\mathrm{MBq}}\right) \times \frac{\text { extrahepatic amount }(\%) \times 250(\mathrm{MBq})}{\text { extrahepatic volume }\left(\mathrm{cm}^{3}\right) \times 1.06\left(\mathrm{~g} / \mathrm{cm}^{3}\right)} .
\end{aligned}
$$

In this formula, $15.87 \mathrm{~mJ} / \mathrm{MBq}$ is the total energy absorbed from the total decay of $1 \mathrm{MBq}$ of ${ }^{166} \mathrm{Ho}, 250 \mathrm{MBq}$ is the activity of injected ${ }^{166} \mathrm{Ho}$ in a scout dose, and a density of soft tissue of $1.06 \mathrm{~g} / \mathrm{cm}^{3}$ was assumed $(13,14)$. All energy was assumed to be absorbed in the extrahepatic deposition location, because the mean penetration of the $\beta$ emission of ${ }^{166} \mathrm{Ho}(2.5 \mathrm{~mm})$ is small, compared with the measured volumes (15).

A safety boundary for the absorbed dose in extrahepatic tissue was reported in the literature by Kao et al. $(16,17)$. After radioembolization with yttrium resin microspheres, the authors quantified the absorbed dose to extrahepatic tissue using PET. An absorbed dose of $18 \mathrm{~Gy}$ was deposited in the stomach of 1 patient, after which no complications occurred. In a second patient, extrahepatic deposition was present in the stomach $(2 \times)$ and duodenum. The patient developed gastritis (stomach), ulceration (stomach), and duodenitis (duodenum) after a mean absorbed dose of 49, 65, and 53 Gy, respectively. For the current study, a 49-Gy boundary was chosen from which toxicity was expected to occur $(16,17)$.

\section{Statistical Analyses}

Data were summarized using descriptive statistics suitable for nonnormal distributed data (medians, range). All statistical analyses were performed in $\mathrm{R}$ (R Foundation for Statistical Computing) (18). 
Confidence intervals for proportions were calculated using the adjusted Wald method and displayed as 95\% confidence intervals (19).

\section{RESULTS}

\section{Phantom Study}

Figure 1 shows the apparent volume for each sphere as a function of threshold level. The thresholds at which the true volumes of the spheres were recovered are denoted by black dots. The optimal threshold level depends on the sphere size. The volume is underestimated for all spheres if a $40 \%$ threshold is used.

Figure 2 shows the $40 \%$ threshold-estimated absorbed doses relative to their true values in the phantom. To estimate the activity in the spheres, the same method was used as for extrahepatic depositions in patients (i.e., without a threshold to avoid underestimating the activity). The counts in a volume including a single sphere were summed and divided by the sum over the total image containing all spheres. The recovered activities, as a percentage of the true, known, injected activities, were (from smallest sphere to largest) $94 \%, 89 \%, 91 \%, 100 \%, 100 \%$, and $101 \%$. Combined with the estimated volumes and with Equation 1 applied, the absorbed dose in each sphere was overestimated with (from smallest to largest sphere) $4 \%, 57 \%, 99 \%, 118 \%, 55 \%$, and $16 \%$.

\section{Patient Population}

Of all the 160 patients undergoing a ${ }^{99 \mathrm{~m}} \mathrm{Tc}-\mathrm{MAA}$ injection between March 2011 and March 2014, 33 patients were identified with extrahepatic deposition of ${ }^{99 \mathrm{~m}} \mathrm{Tc}-\mathrm{MAA}$. Of these 33 patients, the dataset of 1 procedure was not available due to a technical error. In 2 patients, 2 separate depositions were identified, which were treated as separate cases. In total, 32 patients with 34 extrahepatic depositions were included (Fig. 3 provides a flowchart). Baseline characteristics can be found in Table 1. For each of these patients, there were contraindications for treatment due to their extrahepatic deposition, but an additional angiographic procedure allowed treatment in 17 of 32 patients $(53 \%)$.

\section{Extrahepatic Deposition}

Of the infused activity, a median of $1.3 \%$ (range, $0.1 \%-19.5 \%$ ) was located in the extrahepatic depositions. The distribution of

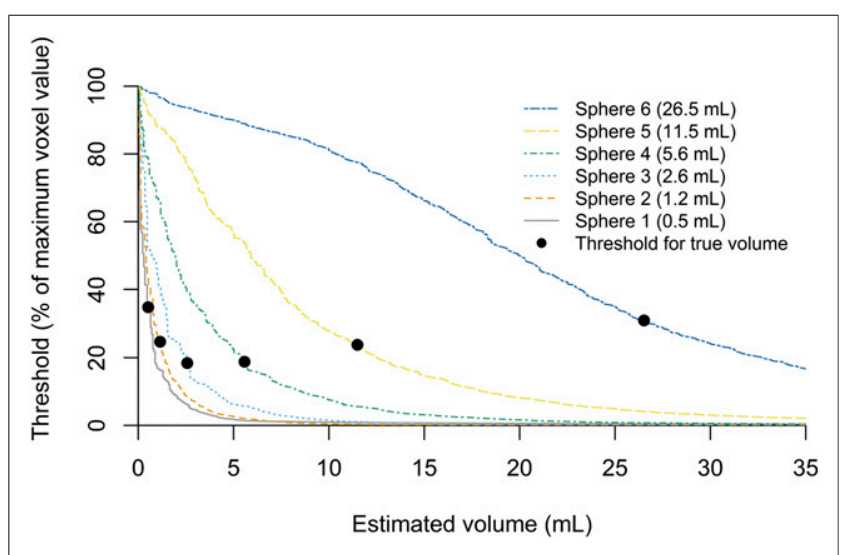

FIGURE 1. Volume estimation in phantom study of 6 spheres of different volumes $(0.5-26.5 \mathrm{~mL})$. Estimate of volume on SPECT is number of all voxels from threshold up to voxel with maximum value, multiplied by voxel volume; it decreases as threshold increases. True, known, volume of each sphere is indicated by black dot. Higher threshold leads to underestimation whereas lower threshold leads to overestimation. For all spheres, a threshold of $40 \%$ leads to underestimation of volume.

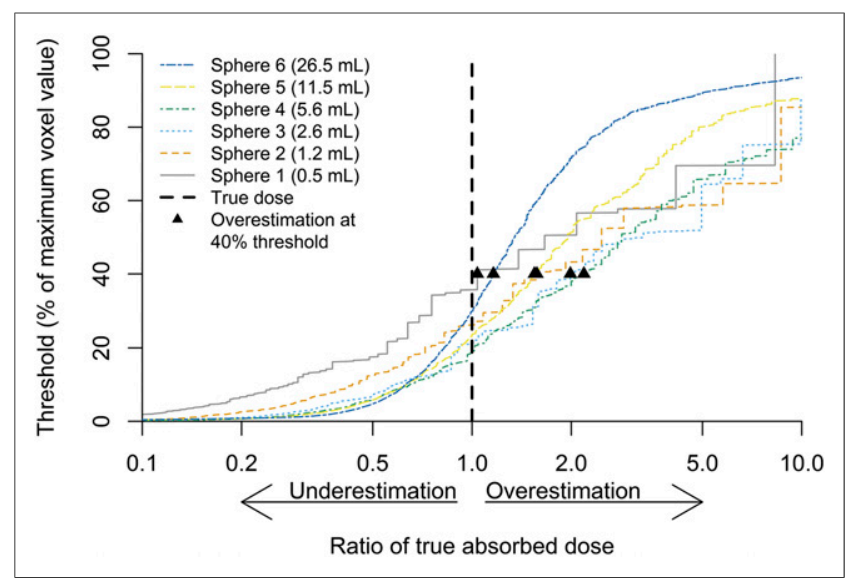

FIGURE 2. Absorbed dose estimation in phantom study using 6 spheres of different volumes $(0.5-26.5 \mathrm{~mL})$. Absorbed dose is displayed as ratio of true absorbed dose in sphere. Threshold needed to estimate true absorbed dose (dotted line) differs per sphere. Absorbed dose is overestimated in all spheres using threshold of $40 \%(\boldsymbol{\Delta})$.

extrahepatic activities was skewed to the right, as can be seen in Figure 4. In the 2 largest outliers, the extrahepatic deposition contained $17.0 \%$ and $19.5 \%$ of the total administered activity. The median-determined volume of the extrahepatic depositions was $6.8 \mathrm{~mL}$ (range, $1.1-42 \mathrm{~mL}$ ). The full dataset is available in Supplemental Table 1 (supplemental materials are available at http://jnm.snmjournals.org).

\section{Theoretic Absorbed Dose After Scout Dose of ${ }^{166} \mathrm{Ho}$}

If a scout dose of $250 \mathrm{MBq}$ of ${ }^{166} \mathrm{Ho}$ had been administered instead of $99 \mathrm{~m}$ Tc-MAA, the median absorbed dose to extrahepatic tissue would have been 6.0 Gy (range, 0.9-374 Gy, right-skewed). Two patients would have received absorbed doses at which complications might have developed: 112 and 374 Gy (Fig. 4B). The largest absorbed dose of $374 \mathrm{~Gy}$ was explained by its large extrahepatic activity $(19.5 \%)$ in a small volume of only $2.1 \mathrm{~mL}$. This patient had liver metastases of a colorectal carcinoma.

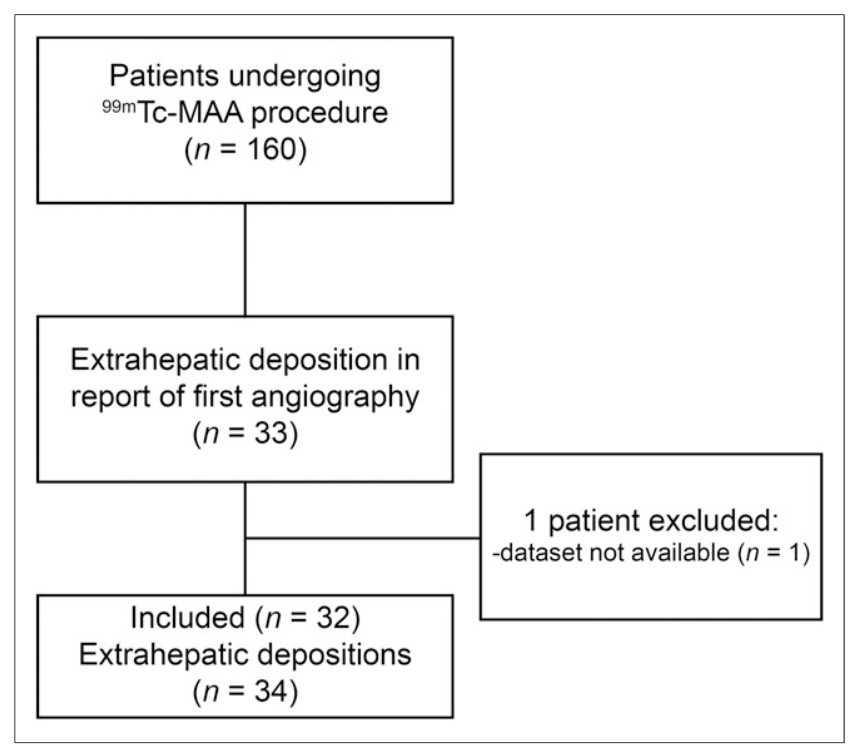

FIGURE 3. Flowchart of study design. 
TABLE 1

Baseline Characteristics

\begin{tabular}{|c|c|}
\hline Characteristic & $\begin{array}{l}n(\%) \text { or median } \\
\text { (range) }\end{array}$ \\
\hline Patients/\# cases & $32 / 34$ \\
\hline \multicolumn{2}{|l|}{ Sex } \\
\hline Male & $25(78 \%)$ \\
\hline Female & $7(22 \%)$ \\
\hline Age $(y)$ & $67(36-80)$ \\
\hline \multicolumn{2}{|l|}{ Primary tumor } \\
\hline Colorectal carcinoma & 19 (59\%) \\
\hline Cholangiocellular carcinoma & $3(9 \%)$ \\
\hline Hepatocellular carcinoma & $3(9 \%)$ \\
\hline Ocular melanoma & $2(6 \%)$ \\
\hline $\begin{array}{l}\text { Adenocarcinoma of unknown } \\
\text { primary }\end{array}$ & $1(3 \%)$ \\
\hline Breast carcinoma & $1(3 \%)$ \\
\hline Gastric carcinoma & $1(3 \%)$ \\
\hline Neuroendocrine tumor & $1(3 \%)$ \\
\hline Ampullary carcinoma & $1(3 \%)$ \\
\hline \multicolumn{2}{|l|}{ Coil embolization } \\
\hline Gastroduodenal and right gastric & $17(53 \%)$ \\
\hline Gastroduodenal & $6(19 \%)$ \\
\hline Gastroduodenal and cystic & $1(3 \%)$ \\
\hline Gastroduodenal and pancreatic & $1(3 \%)$ \\
\hline Gastroduodenal and duodenal & $1(3 \%)$ \\
\hline None & $6(19 \%)$ \\
\hline \multicolumn{2}{|l|}{ No. of injection positions } \\
\hline 1 & $17(53 \%)$ \\
\hline Common & 4 \\
\hline Proper & 11 \\
\hline Right & 1 \\
\hline Replaced left & 1 \\
\hline 2 & $14(44 \%)$ \\
\hline Common + replaced right & 1 \\
\hline Common + replaced left & 1 \\
\hline Right + left & 9 \\
\hline Right + replaced left & 1 \\
\hline Replaced right + left & 1 \\
\hline $\begin{array}{l}\text { Replaced right }+ \text { left (from superior } \\
\text { mesenteric artery) }\end{array}$ & 1 \\
\hline 3 & $1(3 \%)$ \\
\hline Replaced right and selective $(2 x)$ left & 1 \\
\hline
\end{tabular}

He received a first ${ }^{99 m}$ Tc-MAA injection in the proper hepatic artery, after which a large extrahepatic focus was seen on SPECT/CT in the pancreatic region (Fig. 5). Subsequent injections in the left and right hepatic artery, during an additional procedure, showed no extrahepatic deposition, and he finally was treated with resin ${ }^{90} \mathrm{Y}$ microspheres without complications. The other dose, of $112 \mathrm{~Gy}$, would have received less activity in the deposition $(8.7 \%)$ but also in a small volume of $3.1 \mathrm{~mL}$. This

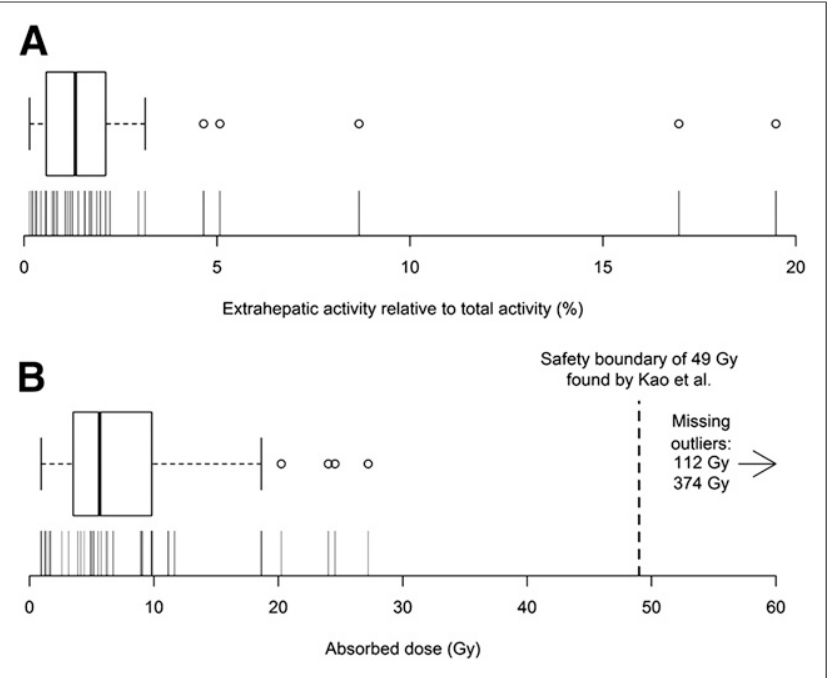

FIGURE 4. (A) Box plot and density plot of extrahepatic activity relative to total activity (liver and extrahepatic deposition). Distribution is right-skewed. Each vertical indicates observation. (B) Box plot and density plot of absorbed dose to extrahepatic tissue from $250-\mathrm{MBq}{ }^{166} \mathrm{Ho}$ scout dose, plotted on log-scale (in Gy). Boundary from which complications occurred in study by Kao et al. of 49 Gy is displayed (17).

patient also had liver metastasis from a colorectal carcinoma. He previously underwent a hemihepatectomy of the right liver. A left hepatic artery and middle hepatic artery of small caliber remained; ${ }^{99 m}$ Tc-MAA was injected in both arteries after coil embolization of the gastroduodenal and right gastric artery. SPECT/CT showed extrahepatic activity in the duodenum (Fig. 6). On repeated angiography, patency of a branch from the proximal gastroduodenal artery was found. Reflux was thought to have occurred because of the small caliber of the vessel after hemihepatectomy. After coil embolization, treatment was successful. The patient showing $17.0 \%$ of extrahepatic activity would not have received a high absorbed dose (only $25 \mathrm{~Gy}$ ) because of its distribution in a relatively large volume of $27.4 \mathrm{~mL}$.

\section{DISCUSSION}

Using a surrogate particle, ${ }^{99 \mathrm{~m}} \mathrm{Tc}-\mathrm{MAA}$, this study shows that the infusion of a scout dose $(250 \mathrm{MBq})$ of therapeutic ${ }^{166} \mathrm{Ho}$ microspheres may theoretically harm extrahepatic tissue by its absorbed dose. In their study, Kao et al. found complications to occur from 49 Gy and upward (16,17). Of 160 patients, 34 cases with extrahepatic deposition were found. In theory, the boundary of 49 Gy was only exceeded in $1.3 \%$ of cases $(2 / 160$; $95 \%$ confidence interval, $0.1 \%-4.7 \%$ ), namely 112 and $374 \mathrm{~Gy}$. In all remaining cases, the absorbed dose would not have exceeded 27.2 Gy (Fig. 4B).

A ${ }^{166} \mathrm{Ho}$ scout dose provides an advantage over ${ }^{99 \mathrm{~m}} \mathrm{Tc}-\mathrm{MAA}$ mostly because it is superior in predicting the absorbed dose by the lungs. Elschot et al. found that planar scintigraphy after a $99 \mathrm{~m}$ Tc-MAA injection vastly overestimated the lung shunt (8). The authors found a median difference of 2.4 Gy (range, 1.0-12.3 Gy) when they compared estimates of the lung-absorbed dose after injection of ${ }^{99 \mathrm{~m} T c-M A A}$ and ${ }^{166} \mathrm{Ho}$ microspheres. The relevance of this finding is shown by the incidence of a high lung shunt fraction on ${ }^{99 \mathrm{~m}} \mathrm{Tc}-\mathrm{MAA}$ SPECT/CT. In the study population, planar 


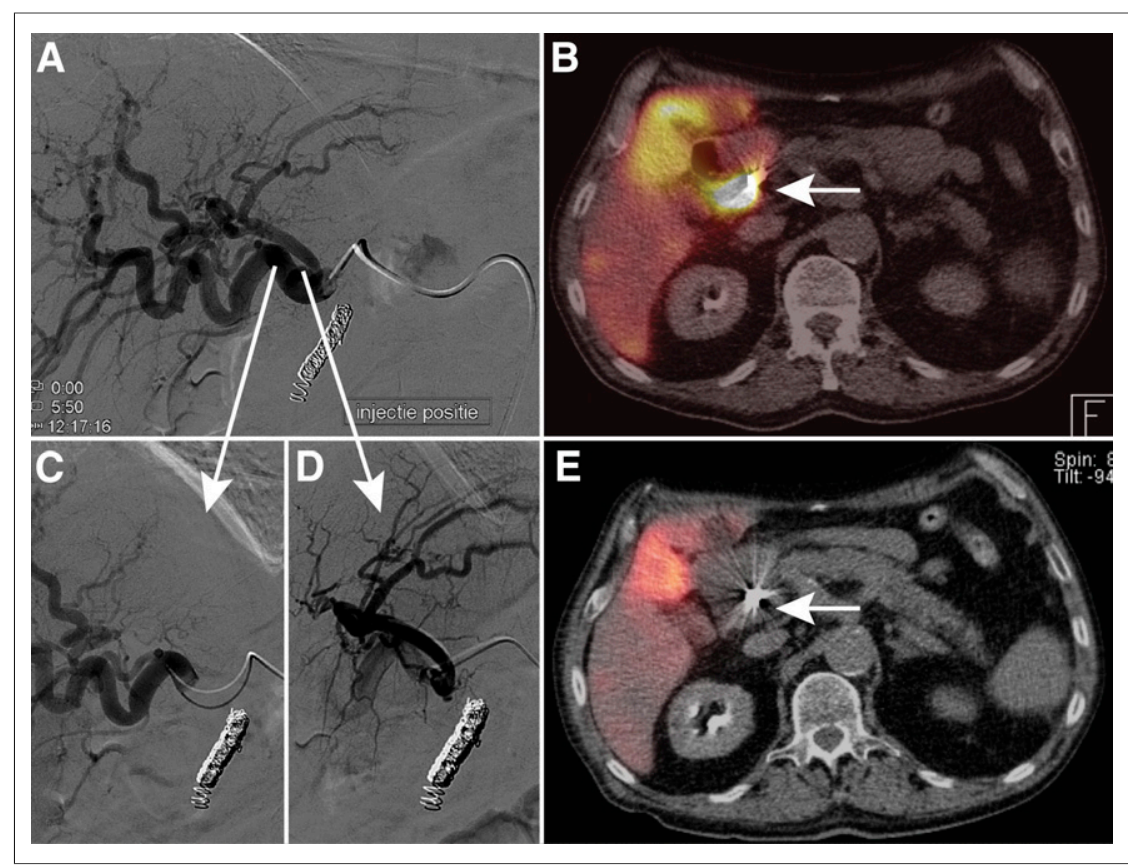

FIGURE 5. Extrahepatic deposition of $8.7 \%$ of administered activity near duodenum after injection in proper hepatic artery (A and B), which was corrected after selective lobar injection in right (C) and left (D) hepatic artery (E). Had $250-\mathrm{MBq}^{166} \mathrm{Ho}$ scout dose been injected, theoretic extrahepatic absorbed dose would have been 112 Gy.

lung shunt fractions were available for 157 of 160 patients. The median lung shunt fraction was $4.9 \%$ (range, $0.3 \%-38.7 \%$ ). Of 157 patients, in 21 patients the lung shunt fraction was greater than $10 \%$
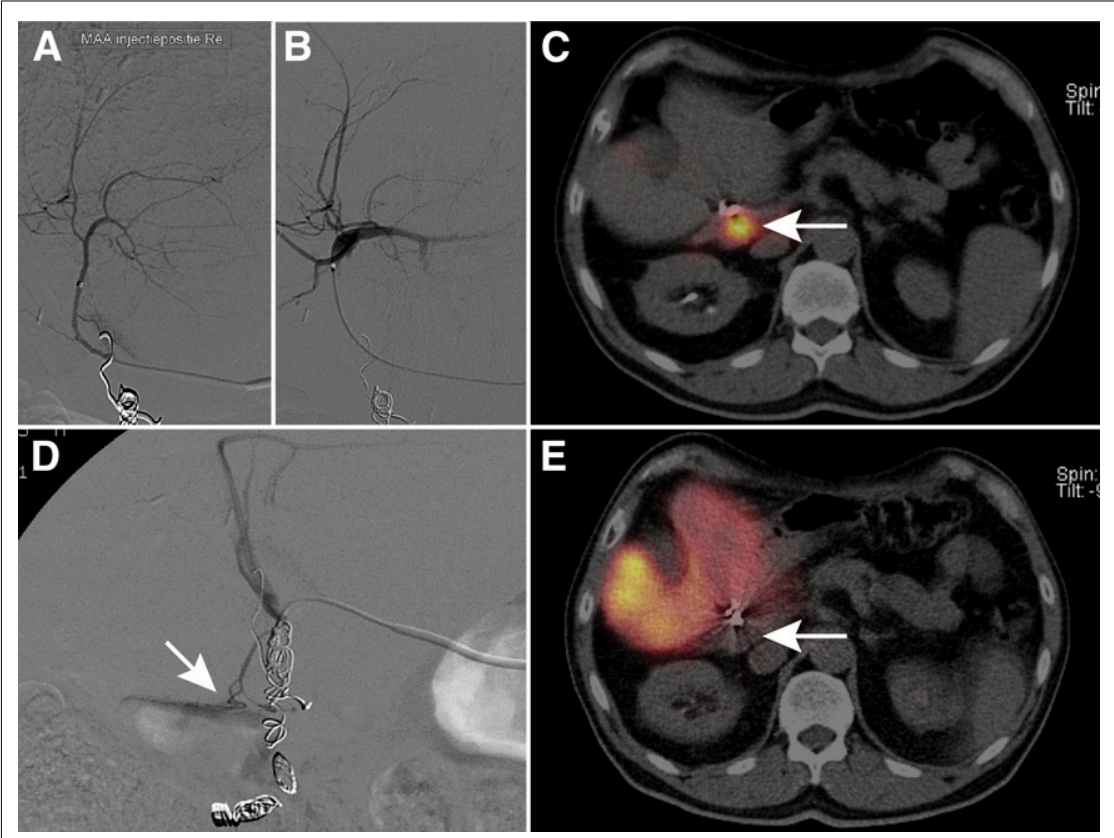

FIGURE 6. Extrahepatic deposition in a patient, who previously received right hemihepatectomy, after injection in right $(\mathrm{A})$ and left $(\mathrm{B})$ hepatic artery. Deposition (C, white arrow) represents $19.5 \%$ of total activity (little activity is seen in liver). During repeated angiography, culprit vessel originated from gastroduodenal artery ( $\mathrm{D}$, white arrow). After coil embolization and single injection in proper hepatic artery, no deposition was seen (E, white arrow). Had $250-\mathrm{MBq}^{166} \mathrm{Ho}$ scout dose been injected, theoretic absorbed dose would have been 374 Gy. (dose reduction advocated), of which 6 were greater than $15 \%$ (further reduction) and of which 3 were greater than $20 \%$ (contraindication). A possible benefit of a more accurate estimation of the lung shunt by ${ }^{166}$ Ho microspheres might have been relevant in $13 \%(21 / 157)$ of the patients. The study population was predominantly composed of patients with colorectal liver metastases, and it has been suggested that these patients have a lower lung shunt fraction than patients with other primary malignancies (20). Powerski et al. reported on a population of 233 patients (29\% colorectal carcinoma, 27\% hepatocellular carcinoma) and found lung shunt fractions to differ between tumor types, showing that patients with hepatocellular carcinoma had higher lung shunt fractions (21). Incidences of a high lung shunt fraction will vary between institutions as patient populations vary. Gaba et al. reported an incidence of a high lung shunt $(>10 \%)$ in $40 \%$ of patients; $50 \%$ of their population of 141 patients consisted of patients with hepatocellular carcinoma (22). A better estimator of the lung shunt would thus be relevant for clinical practice.

Additionally, it is hypothesized that the predictive value of a ${ }^{166} \mathrm{Ho}$ microsphere scout dose is superior to a ${ }^{99 m}$ Tc-MAA scout dose, because the microspheres of the scout and therapeutic dose are identical. In that case, treatment could be optimized by increasing the radioactive dose in some arteries (e.g., with a high tumorto-nontumor ratio) and by limiting it in others, possibly leading to a higher efficacy and lower toxicity. Imaging of both ${ }^{99 \mathrm{~m}} \mathrm{Tc}-\mathrm{MAA}$ and ${ }^{166} \mathrm{Ho}$ can be performed by SPECT/CT. ${ }^{99 \mathrm{~m} T c}$ emits $140-\mathrm{keV} \gamma$ rays with a radiation abundance of $89 \%$, whereas ${ }^{166} \mathrm{Ho}$ emits $81-\mathrm{keV} \gamma$ rays with an abundance of $7 \%$ (23). Although spatial resolution, contrast recovery, and sensitivity are worse for ${ }^{166} \mathrm{Ho}$ than ${ }^{99 \mathrm{~m}} \mathrm{Tc}-\mathrm{MAA}$, injection of $250 \mathrm{MBq}$ of ${ }^{166} \mathrm{Ho}$ microspheres is sufficient to provide images that allow for the evaluation of lung shunt and extrahepatic deposition (24,25). Another benefit of ${ }^{166} \mathrm{Ho}$ microspheres, and a principal reason for their development (26), is the possibility of their multimodal detection: an 81-keV photopeak for nuclear imaging (24), high magnetic susceptibility for MR imaging (27), and a high mass attenuation coefficient for CT imaging (28). The most promising modality, MR imaging, could enable MR-guided treatment, as has been performed ex vivo in rabbits (29).

The absorbed doses presented in this study should be considered with care, as they are most likely dose overestimations (4\%-118\% in the phantom study). The 
dose calculations are dependent on the activity and volume estimations. The latter depends on the chosen threshold, as seen in Figure 1. The accuracy of SPECT is influenced by detrimental breathing effects, limited resolution, scatter, and septal penetration. Furthermore, volume estimations of small volumes $(<1 \mathrm{~mL})$ may be less precise, which can be seen in Figure 1 by the density of the curves for the smaller spheres. Our phantom study consisted of homogeneously filled spheres, whereas extrahepatic depositions may be more cylindric and consist of heterogeneous clusters of microspheres. Because our measurements could not be exact, an underestimation of the theoretic absorbed dose had to be avoided, as this could lead us to conclude that a ${ }^{166} \mathrm{Ho}$ scout dose was safe while it might not be. In our phantom study, we showed that a threshold of $40 \%$ will underestimate the volume (Fig. 1) and thereby overestimate the absorbed dose (Fig. 2).

The preliminary published threshold of 49 Gy is based on 1 publication, a case series in which extrahepatic depositions from radioembolization were quantified. This threshold might be an underestimation, as the same absorbed dose in a different volume might lead to a different clinical outcome, for example, high doses in a large volume might be more toxic than in a small volume. It might also be an overestimation, as the threshold on external radiotherapy was found to be much lower (30). Although absorbed doses from external radiotherapy are not comparable to radioembolization (due to differences in biologic effective doses) and literature on complications of the stomach and small bowels is scant, data on external radiotherapy do provide a clue. A risk of 5\% for toxicities within $5 \mathrm{y}$ was estimated after partial stomach or small-bowel irradiation with $50 \mathrm{~Gy}$. However, stereotactic body radiation therapy achieves higher absorbed doses per fraction and might be more comparable to radioembolization; Kavanagh et al. advise to minimize the maximum absorbed point dose in $5 \mathrm{~mL}$ of stomach to less than $30 \mathrm{~Gy}$ and minimize the amount of small-bowel volume irradiation with more than 12.5 Gy to less than $30 \mathrm{~mL}$. A study by Streitparth et al., investigating 1-time high-dose-rate brachytherapy of liver malignancies, found a threshold absorbed dose of $11 \mathrm{~Gy}$ in a 1-mL volume of stomach wall for gastric toxicities (31). The authors noted that findings from high-dose-rate brachytherapy (irradiation time, 20-40 min) vary from external radiotherapy, as the latter is probably less toxic. For ${ }^{166} \mathrm{Ho}$, with a half-life of $26.8 \mathrm{~h}, 90 \%$ of the dose is deposited over $89 \mathrm{~h}$. In our study, the large difference between the outliers (112 and 374 Gy) and the highest safe absorbed dose (27.2 Gy) shows that the safety threshold of 49 Gy need not be very accurate for this study to draw the same conclusions. If accurate dosimetry studies on radioembolization become available, the data from this study can be reevaluated (all data are available in Supplemental Table 1).

Extrahepatic deposition after radioembolization treatment can cause ulceration or inflammation, probably because of both embolization and radiation damage. We investigated only the latter. An animal study in 9 pigs by Bilbao et al. (2) showed that nonradioactive microspheres only induce ulceration when aggregated, and embolization of small distal vessels alone does not cause ulceration. Blood flow was regenerated by the appearance of new vessels or recanalization of occluded vessels. A report by Ogawa et al. of 3 patients who developed gastroduodenal complications after hepatic radioembolization suggested that the changes in the gastroduodenal region were similar in histologic appearance and timing to radiation-induced damage (3). Murthy et al. noted that some ulcers do not contain microspheres on histopathologic examination and posed another causative mechanism: Bremsstrahlung of adjacent liver tissue treated with radioembolization (4). After ex- trahepatic deposition, it seems likely embolization has an additional negative effect, the extent of which is unknown. Embolization is also dependent on different variables, including microsphere composition and size. It is therefore not incorporated in this study.

An analysis of the causes of extrahepatic depositions is beyond the scope of this study. However, our cohort is in accordance with findings by Lam et al. (32), who found that a proximal injection can be a cause; we found ${ }^{99 m}$ Tc-MAA was often injected in a proximal, whole-liver $(47 \%, 15 / 32)$ fashion. Another factor the authors mentioned - that is, stasis during injection — did not occur, because 99mTc-MAA has little embolic effect. Paradoxically, we found that most patients underwent prophylactic occlusion of one or more arteries, even though coil embolization is no longer routinely performed in our center. Unfortunately, after introduction of C-arm CT in our institute, extrahepatic depositions still occurred. A preventive effect hereof could not be tested for in the present analysis. The high incidence of extrahepatic activity on 99mTc-MAA SPECT/CT (33/ 160 patients) may have been related to a learning curve (e.g., administration in the proper hepatic artery vs. more selective administration) and the late introduction of technical innovations (e.g., C-arm $\mathrm{CT}$ ). When the incidence of extrahepatic activity decreases because of either, the relative benefit of a ${ }^{166} \mathrm{Ho}$ scout dose increases, as it has superior lung shunt estimation and probably allows for a more accurate dosimetry-based treatment planning. Not all extrahepatic deposition could be corrected, and only $53 \%$ of patients were ultimately treated with radioembolization.

A ${ }^{166} \mathrm{Ho}$ scout dose of $250 \mathrm{MBq}$ was used for assessment, because it is currently used in clinical studies. By decreasing this activity, the absorbed dose can be decreased. The current activity was chosen after several preclinical studies to find a balance between toxicity and detectability. The potential lung dose of a ${ }^{166} \mathrm{Ho}$ scout dose is, for example, far below a clinically relevant absorbed dose of $30 \mathrm{~Gy}$ (33). To reduce this activity would render it safer but reduce its detectability.

In this study, the incidence of an extrahepatic deposition of a ${ }^{166} \mathrm{Ho}$ scout dose that is high enough to cause complications after injection is low $(<2 \%)$. The high absorbed doses found in this theoretic analysis do not necessarily translate to complications in practice. From a risk-benefit perspective, an injection of ${ }^{99 \mathrm{~m}} \mathrm{Tc}-$ MAA is preferred over a ${ }^{166} \mathrm{Ho}$ scout dose in the absence of significant benefits. However, a better estimation of the lung dose (and anticipated treatment individualization) seems to outweigh the risk. Additionally, improved pretreatment imaging and additional C-arm CT imaging may decrease the risk and severity of extrahepatic depositions $(5,34)$. The workup for ${ }^{166} \mathrm{Ho}$ radioembolization in 2 clinical studies that recently started recruitment in our center has now been changed. Digital subtraction angiography and $\mathrm{C}$-arm CT are always performed from every injection position, and a ${ }^{99 m}$ Tc-MAA scout dose was replaced by a ${ }^{166}$ Ho scout dose (Surefire Infusion System vs. Standard Microcatheter Use During Holmium166 Radioembolization study, NCT02208804; HEPAR Plus study, NCT02067988). The safety a ${ }^{166} \mathrm{Ho}$ scout dose is continuously evaluated in these prospective clinical trials.

\section{CONCLUSION}

Theoretic analysis of the potential risk of a $250-\mathrm{MBq}{ }^{166} \mathrm{Ho}$ scout dose resulted in a low incidence $(1.3 \%$; $95 \%$ confidence interval, $0.1 \%-4.7 \%)$ of potentially harmful extrahepatic deposition. Because its clinical benefits, which include a more accurate estimate of the lung shunt, seem to outweigh its potential risk, the 
${ }^{99 m}$ Tc-MAA injection was replaced by a ${ }^{166} \mathrm{Ho}$ scout dose in ongoing clinical trials on ${ }^{166} \mathrm{Ho}$ radioembolization. Its safety is evaluated further in clinical practice.

\section{DISCLOSURE}

The costs of publication of this article were defrayed in part by the payment of page charges. Therefore, and solely to indicate this fact, this article is hereby marked "advertisement" in accordance with 18 USC section 1734. Marnix G.E.H. Lam is advisor for BTG and Bayer Healthcare and speaker for Sirtex Inc. No other potential conflict of interest relevant to this article was reported.

\section{REFERENCES}

1. Seidensticker R, Denecke T, Kraus P, et al. Matched-pair comparison of radioembolization plus best supportive care versus best supportive care alone for chemotherapy refractory liver-dominant colorectal metastases. Cardiovasc Intervent Radiol. 2012;35:1066-1073.

2. Bilbao JI, de Martino A, de Luis E, et al. Biocompatibility, inflammatory response, and recannalization characteristics of nonradioactive resin microspheres: histological findings. Cardiovasc Intervent Radiol. 2009;32:727-736.

3. Ogawa F, Mino-Kenudson M, Shimizu M, Ligato S, Lauwers GY. Gastroduodenitis associated with yttrium 90-microsphere selective internal radiation: an iatrogenic complication in need of recognition. Arch Pathol Lab Med. 2008;132:1734-1738.

4. Murthy R, Brown DB, Salem R, et al. Gastrointestinal complications associated with hepatic arterial yttrium-90 microsphere therapy. J Vasc Interv Radiol. 2007;18: 553-561.

5. Heusner TA, Hamami ME, Ertle J, et al. Angiography-based C-arm CT for the assessment of extrahepatic shunting before radioembolization. Rofo. 2010;182:603-608.

6. Lau WY, Leung TW, Ho S, et al. Diagnostic pharmaco-scintigraphy with hepatic intraarterial technetium-99m macroaggregated albumin in the determination of tumour to non-tumour uptake ratio in hepatocellular carcinoma. $\mathrm{Br} J$ Radiol. 1994;67:136-139.

7. Smits ML, Nijsen JF, van den Bosch MA, et al. Holmium-166 radioembolisation in patients with unresectable, chemorefractory liver metastases (HEPAR trial): a phase 1, dose-escalation study. Lancet Oncol. 2012;13:1025-1034.

8. Elschot M, Nijsen JFW, Lam MGEH, et al. ${ }^{99 m}$ Tc-MAA overestimates the absorbed dose to the lungs in radioembolization: a quantitative evaluation in patients treated with ${ }^{166}$ Ho-microspheres. Eur J Nucl Med Mol Imaging. 2014;41:19651975.

9. Boellaard R, Krak NC, Hoekstra OS, Lammertsma A. Effects of noise, image resolution, and ROI definition on the accuracy of standard uptake values: a simulation study. J Nucl Med. 2004;45:1519-1527.

10. Kennedy A, Nag S, Salem R, et al. Recommendations for radioembolization of hepatic malignancies using yttrium-90 microsphere brachytherapy: a consensus panel report from the radioembolization brachytherapy oncology consortium. Int J Radiat Oncol Biol Phys. 2007;68:13-23.

11. Bol GH, Kotte AN, van der Heide UA, Lagendijk JJ. Simultaneous multi-modality ROI delineation in clinical practice. Comput Methods Programs Biomed. 2009;96:133-140.

12. Sabet A, Ahmadzadehfar H, Muckle M, et al. Significance of oral administration of sodium perchlorate in planning liver-directed radioembolization. $\mathrm{J} \mathrm{Nucl} \mathrm{Med}$. 2011;52:1063-1067.

13. Vente MAD, Nijsen JFW, de Wit TC, et al. Clinical effects of transcatheter hepatic arterial embolization with holmium-166 poly(L-lactic acid) microspheres in healthy pigs. Eur J Nucl Med Mol Imaging 2008;35:1259-1271.
14. International Commission on Radiation Units and Measurements (ICRU). Tissue Substitutes in Radiation Dosimetry and Measurement. ICRU report 44. Bethesda, MD: ICRU; 1989.

15. Bult W, Kroeze SG, Elschot M, et al. Intratumoral administration of holmium166 acetylacetonate microspheres: antitumor efficacy and feasibility of multimodality imaging in renal cancer. PLOS ONE. 2013;8:e52178.

16. Kao Y-H, Steinberg JD, Tay Y-S, et al. Post-radioembolization yttrium-90 PET/ CT: part 1-diagnostic reporting. EJNMMI Res. 2013;3:56.

17. Kao Y-H, Steinberg JD, Tay Y-S, et al. Post-radioembolization yttrium-90 PET/ CT: part 2-dose-response and tumor predictive dosimetry for resin microspheres. EJNMMI Res. 2013;3:57.

18. R: A language and environment for statistical computing. The R Foundation website. http://www.r-project.org. Accessed April 28, 2015.

19. Agresti A, Coull BA. Approximate is better than "exact" for interval estimation of binomial proportions. Am Stat. 1998;52:119-126.

20. Gates V, Salem R. Reply to "hepatic radioembolization as a true single-session treatment." J Vasc Interv Radiol. 2014;25:1144-1146.

21. Powerski MJ, Erxleben C, Scheurig-Münkler C, et al. Hepatopulmonary shunting in patients with primary and secondary liver tumors scheduled for radioembolization. Eur J Radiol. 2015;84:201-207.

22. Gaba RC, Zivin SP, Dikopf MS, et al. Characteristics of primary and secondary hepatic malignancies associated with hepatopulmonary shunting. Radiology. 2014;271:602-612.

23. Elschot M, Nijsen JFW, Dam AJ, de Jong HWAM. Quantitative evaluation of scintillation camera imaging characteristics of isotopes used in liver radioembolization. PLOS ONE. 2011;6:e26174.

24. Elschot M, Smits ML, Nijsen JF, et al. Quantitative Monte Carlo-based holmium-166 SPECT reconstruction. Med Phys. 2013;40:112502.

25. de Wit TC, Xiao J, Nijsen JF, et al. Hybrid scatter correction applied to quantitative holmium-166 SPECT. Phys Med Biol. 2006;51:4773-4787.

26. Mumper RJ, Ryo UY, Jay M. Neutron-activated holmium-166-poly (L-lactic acid) microspheres: a potential agent for the internal radiation therapy of hepatic tumors. J Nucl Med. 1991;32:2139-2143.

27. van de Maat GH, Seevinck PR, Elschot M, et al. MRI-based biodistribution assessment of holmium-166 poly(L-lactic acid) microspheres after radioembolisation. Eur Radiol. 2013;23:827-835.

28. Seevinck PR, Seppenwoolde J-H, de Wit TC, et al. Factors affecting the sensitivity and detection limits of MRI, CT, and SPECT for multimodal diagnostic and therapeutic agents. Anticancer Agents Med Chem. 2007;7:317-334.

29. Nijsen JF, Seppenwoolde J-H, Havenith T, Bos C, Bakker CJ, van het Schip AD. Liver tumors: MR imaging of radioactive holmium microspheres-phantom and rabbit study. Radiology. 2004;231:491-499.

30. Kavanagh BD, Pan CC, Dawson LA, et al. Radiation dose-volume effects in the stomach and small bowel. Int J Radiat Oncol Biol Phys. 2010;76: S101-S107.

31. Streitparth F, Pech M, Böhmig M, et al. In vivo assessment of the gastric mucosal tolerance dose after single fraction, small volume irradiation of liver malignancies by computed tomography-guided, high-dose-rate brachytherapy. Int J Radiat Oncol Biol Phys. 2006;65:1479-1486.

32. Lam MG, Banerjee S, Louie JD, et al. Root cause analysis of gastroduodenal ulceration after yttrium-90 radioembolization. Cardiovasc Intervent Radiol. 2013;36:1536-1547.

33. Dezarn WA, Cessna JT, DeWerd LA, et al. Recommendations of the American Association of Physicists in Medicine on dosimetry, imaging, and quality assurance procedures for ${ }^{90} \mathrm{Y}$ microsphere brachytherapy in the treatment of hepatic malignancies. Med Phys. 2011;38:4824-4845.

34. van den Hoven AF, van Leeuwen MS, Lam MG, van den Bosch MA. Hepatic arterial configuration in relation to the segmental anatomy of the liver; observations on MDCT and DSA relevant to radioembolization treatment. Cardiovasc Intervent Radiol. 2015;38:100-111. 